\title{
EDITORIAL
}

nature

cell biology

\section{Changing gear}

\section{Is scientific progress being stifled by a lack of support for researchers who aim to change research directions?}

At a time when cell biologists are exposed more than ever to diverse fields of research, it is notable that we are not seeing a parallel increase in researchers moving into new areas. There are certainly compelling examples of cell biologists who have successfully maintained more than one research focus or shifted the direction of their laboratory entirely — but not as many as one might expect. Perhaps people really do have a healthy obsession with their particular research focus, but is there also a fear of failure that is being compounded by the current funding and publishing process?

When applying for your first independent position, there may be a temptation to stick with the familiar. Taking the road less travelled and establishing your own niche is no doubt a daunting prospect but one that should be encouraged. Indeed, sticking with your old field can at times make it harder to extricate yourself from the shadow of a famous mentor. Either way, it takes time to establish an independent reputation and contacts within the field - so why shift areas and start all over again, once a certain degree of kudos has been achieved? Well, your research is likely to result in discoveries that could lead you into a new field. At that point "you have a choice either to pursue it or drop it. It can be a matter of having the confidence to go for it - to take the risk", says Ian Macara at the University of Virginia, who has successfully worked on both nuclear transport and polarity for many years.

One danger of pursuing multiple strands of research is that you run the risk of spreading yourself too thin. And it would be a worrying sign if someone felt the urge to drop a project at every sign of pastures new. That said, it is important that researchers remain sufficiently flexible to follow enticing leads; this is true for both the question itself and the approaches that one uses to tackle it. As the 'activation energy' needed to shift research gears is high, support must be in place to facilitate a rational move into new territory.

One issue is that grant review panels often look for evidence of preliminary data. This has in fact led to a culture of submitting grants for half-finished projects and using them for 'the next step'. The recent announcement of proposed changes to the NIH peer-review system may provide a step in the right direction: more support is to be given to new ideas and higher-risk science, and schemes such as the Pioneer Award, requiring that "the proposed research must reflect ideas substantially different from those already being pursued in the investigator's laboratory or elsewhere" (http://nihroadmap.nih.gov/pioneer/ and Science 319, 1169; 2008).
Other funding bodies also need to be more encouraging of people branching out, and to de-emphasize a publication track record in the proposed area of investigation and preliminary data. What should be assessed are the rationale and the promise of the move. Max Planck and Howard Hughes are good examples of an environment that encourages this. Wieland Huttner at the Max Planck Institute in Dresden switched the focus of his laboratory entirely, from neurosecretory vesicles to neural-progenitor cell biology, but did so over the course of several years. As Huttner explains, "Neurogenesis was a long-term interest of mine, but it took time to produce our first decent results and I felt it was important to keep up my old field to guarantee grant support for the lab. It was only when I joined the Max Planck Society and relied less on grants that I could completely make the switch".

But it does not only depend on funding: some fields appear to be harder to break into than others. Particularly in smaller fields, there can be a sense of ownership by dominant individuals. In such cases, it falls to journal editors to make strong decisions in the face of reviewer opposition to new ideas. The editorial premise of all the Nature journals is very much to encourage the unexpected, but ultimately, the attitudes of reviewers to new ideas will influence whether a field moves forward or not. As a referee, ask yourself whether your field-specific views constrain your judgement of a study. As an author, a certain level of perseverance is needed. As Macara says, "It is important to be patient. If you keep doing good work, you will eventually be accepted by that community".

It may often be wise to initiate collaborations to enter new territory. Indeed, one approach that has already been adopted by new institutes, such as Howard Hughes' Janelia Farm, encourages diversity by fostering interdisciplinary collaborations. Another successful example is the Max Planck Institute of Molecular Cell Biology and Genetics in Dresden, which has promoted crosstalk between developmental and cell biology. The premise of such centres is that physical proximity encourages people from different groups to mix and mingle (Nature 451, 872; 2008). It may be argued, however, that the very people who will thrive in such an environment would do equally well wherever they are. "In the end," says Huttner, "it is the individual scientist who makes the choices and good people will seek out collaborators when necessary". Importantly, cross-disciplinary institutes do not really take care of the boundaries experienced at the level of the individual laboratory and long-term support should be in place to encourage people to pursue new directions even within their discipline.

No doubt some of the best research is done by those who appreciate a particular system in all its depth and complexity; however, a fresh view can provide clarity. Exploring new areas may be risky for the individual but there is no question that it drives overall research progress. So if it is that 'rainy day' question that keeps your brain ticking into the early hours, the advice from other trailblazers would be have courage and take that leap.

Connotea.org/user/ncb/tag/changing\%20gear 